\title{
Research on Image Processing Technique for Small Targets in Large Field of View Based on Linear CCD
}

\author{
Liqun Zhang ${ }^{1, a}$, Ping Song ${ }^{1, b}$ and Chuangbo $\mathrm{Hao}^{1, \mathrm{c}}$ \\ ${ }^{1}$ Beijing Institute of Technology, Beijing 100081, China

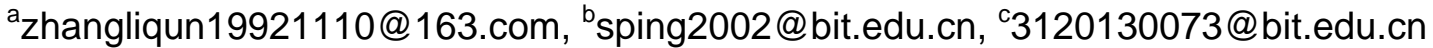

Keywords: Large field of view, small target detection, image enhancement, median filtering, Gabor.

\begin{abstract}
Aiming at the problem of difficulty in detection of small targets in large field of view, an image processing algorithm is proposed to detect small targets in large field of view. The algorithm first performs image preprocessing using grayscale transformation and median filtering, then Gabor matched filtering and threshold segmentation are used to obtain binary image. For getting the position of the small target in target image, the centroid algorithm is presented. Finally, the algorithm is validated by practical experiments. The results show that the algorithm can effectively detect small targets.
\end{abstract}

\section{Introduction}

In recent years, a large field of view formed by linear array CCD cameras is often used to capture high-speed small target to perform target parameter test [1]. Getting the position of small targets in linear array image is an important problem. The high-speed small target only occupies a few pixels on the imaging plane, and appears as a speckle in the line array image. Under the influence of atmospheric transmission and sky background light, the contrast of the target image is very low, and the small target detection is much more difficult than surface target and large target detection[2].

At present, image processing methods for small targets include frame difference[3], wavelet domain analysis background suppression[4], morphological Top Hat filtering[5], Butterworth high-pass filtering[6], two-dimensional least mean square filtering[7], etc. However, these methods are area array image processing methods, they can not be directly applied to linear array images, and the detection is poor when image contrast is low. Based on the analysis of the image characteristics, this paper proposes a new small target detection algorithm for the linear array image under low contrast conditions. The algorithm is implemented in MATLAB. Finally, the algorithm is used to process the small target image in large field of view, which proves the effectiveness of the algorithm. All manuscripts must be in English, also the table and figure texts, otherwise we cannot publish your paper. Please keep a second copy of your manuscript in your office. When receiving the paper, we assume that the corresponding authors grant us the copyright to use the paper for the book or journal in question. Should authors use tables or figures from other Publications, they must ask the corresponding publishers to grant them the right to publish this material in their paper.

\section{Analysis of Small Target Image}

An image collected by the linear array CCD camera can be expressed as[8]:

$$
I(x, y)=I_{T}(x, y)+I_{B}(x, y)+n(x, y)
$$

In the formula, $I(x, y)$ is the gray value of the image at the pixel coordinate $(x, y), I_{T}(x, y)$ is the gray value of the target, $I_{B}(x, y)$ is the gray value of the background, $n(x, y)$ is the gray value of noise. The background of the image is the sky background collected by the CCD camera in the outdoor test. It is a scene with large area changing gently. The noise is the random noise generated by the linear CCD in the process of image acquisition. The high-speed small target flies through the large field of view of the camera. In the linear array mosaic image, the target appears as a nearly vertical patchy 
shape, and the edges show an approximate Gaussian distribution. The difference between the target gray value and the sky background gray value is not obvious, and the contrast is low.

\section{Method of Image Processing}

\subsection{Image Enhancement}

Grayscale transformation can increase the contrast of the image, and it can be expressed as:

$$
I_{o}=l_{\text {out }}+\left(h_{\text {out }}-l_{\text {out }}\right) \frac{I-l_{\text {in }}}{h_{\text {in }}-l_{\text {in }}}
$$

Where $l_{i n}, h_{i n}$ is the minimum and maximum gray value of the image before the image is changed, and $l_{\text {out }}, h_{\text {out }}$ is the minimum and maximum gray value of the image after grayscale adjustment.

\subsection{Median Filtering}

In order to filter the random noise effectively and avoid the small target in the image being filtered,this paper uses a small kernel median filtering method to process the image. Median filtering can reduce the isolated noise point of the image. Small kernel median filtering belongs to non-linear spatial filtering, and it can effectively preserve the detail information in the image while removing the isolated noise pixels in the image. The basic model of median filtering is:

$$
\hat{I}(x, y)=\underset{(i, j) \in B}{\operatorname{median}}[I(i, j)]
$$

Where $B$ represents the neighborhood of pixels, $I(x, y)$ is the origin image, $\hat{I}(x, y)$ is the filtered image.

\subsection{Gabor Matched Filtering and Image Binarizition}

The frequency and direction expression of Gabor kernel is close to the human visual system, so it is commonly used in texture representation and description. From the analysis of the image characteristics, we can see that the small target has obvious texture characteristics in the linear array mosaic image. In this paper, Gabor convolution kernel with small target characteristics is designed to perform matched filtering, and finally the target position is detected.

In the space domain, a two-dimensional Gabor kernel function is product of a sinusoidal plane wave and a Gaussian kernel function. It has the properties of optimal localization in the spatial domain and frequency domain, so it can describe the local structure information which is corresponding to spatial frequency, spatial location and direction-selective. Gabor kernel function $[9,10,11]$ can be expressed as:

$$
G(x, y)=\frac{1}{2 \pi \sigma^{2}} \exp \left(-\frac{x^{2}+y^{2}}{2 \sigma^{2}}\right) \cos (A x)
$$

Where $\sigma$ is the standard deviation of Gaussian function. $A$ is texture frequency, which makes the Gabor kernel function having frequency selection characteristics. The image being matched filtered can be expressed as:

$$
I_{g}(x, y)=G(x, y) \otimes I_{o}
$$

According to the principle of the matched filter, the target texture grayscale and the convolution kernel texture grayscale are cross-correlated. When the similarity is bigger, the output value is larger. Finally, a threshold is used to obtain the target region which satisfies the threshold condition. That is, the image is binarized. It can be expressed as:

Where $\varsigma$ is the threshold for use.

$$
I_{\mathrm{go}}(x, y)=\left\{\begin{array}{lll}
1 & \text { if } & I_{g}(x, y)>\varsigma \\
0 & \text { if } & I_{g}(x, y) \leq \varsigma
\end{array}\right.
$$

During matched filtering, the algorithm rotates the kernel function from $-10^{\circ}$ to $10^{\circ}$ in 5 steps. Figure 1 shows the Gabor kernel function grayscale in different directions when the standard deviation $\sigma=30^{\circ}$, frequency $A=50$. 


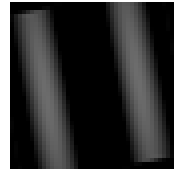

(a) $\theta=-10^{\circ}$

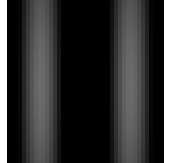

(b) $\theta=0^{\circ}$

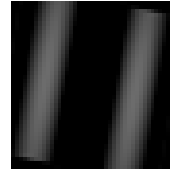

(c) $\theta=10^{\circ}$

Fig.1 Matched filter distribution in different directions

\subsection{Centroid Algorithm}

The principle of the centroid algorithm is based on the binary image, sample the part above the binarization threshold in high frequency, and then calculate the area center to replace the energy center. Moment of function is applicable to shape analysis. The $(p+q)$ order moment of binary function $f(x, y)$ is defined as follows:

$$
m_{p q}=\sum_{i=0}^{M-1} \sum_{j=0}^{N-1} i^{p} j^{q} f(i, j)
$$

0 order of function is the sum of grayscale image. $m_{00}$ of binary image represents the area of object. The center of gravity of object $(i, j)$ can be calculated as follows:

$$
\left\{\begin{array}{l}
\bar{i}=m_{10} / m_{00}=\sum_{i=0}^{M-1} \sum_{j=0}^{N-1} \text { if }(i, j) / \sum_{i=0}^{M-1} \sum_{j=0}^{N-1} f(i, j) \\
\bar{j}=m_{01} / m_{00}=\sum_{i=0}^{M-1} \sum_{j=0}^{N-1} j f(i, j) / \sum_{i=0}^{M-1} \sum_{j=0}^{N-1} f(i, j)
\end{array}\right.
$$

For binary images, the gray value is only 0 and 1 , so the center of gravity is the center of area. In this paper, $f(i, j)$ is the gray value of the pixel in row $i$, column $j . \bar{i}$ is the row of the pixel position where the target is in linear CCD, and $\bar{j}$ is the column.

\section{Test Results and Analysis}

In the practical experiment, $10 \mathrm{~m} \times 10 \mathrm{~m}$ large field of view was formed by the intersection of two linear array CCD cameras, the velocity of the small targrt is $600 \sim 800 \mathrm{~m} / \mathrm{s}$, and the diameter is about $8 \mathrm{~mm}$. The image processing algorithm is validated in MATLAB. The results are shown in Figure 2 and Figure 3. (a)is the original image,(b) is a contrast enhancement image after grayscale adjustment, (c) is an image after median filtering processing, and (d) is a Gabor matched filtering image, (e) is a binary image. It can be seen that, after contrast enhancement, the contrast of the object in the image is significantly improved, but the corresponding random noise is also enhanced. After median filtering, the random noise is obviously suppressed, which can be used as the input image of Gabor matched filtering. After Gabor matched filtering, the position which is similar to the texture feature of the target is highlighted,After image binarization, we can clearly see the small target. The results show that the method proposed in this paper can effectively detect moving small targets in low contrast images.

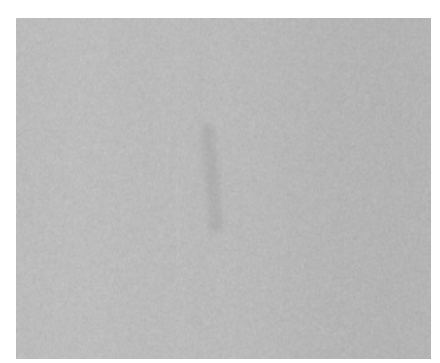

(a)Original image

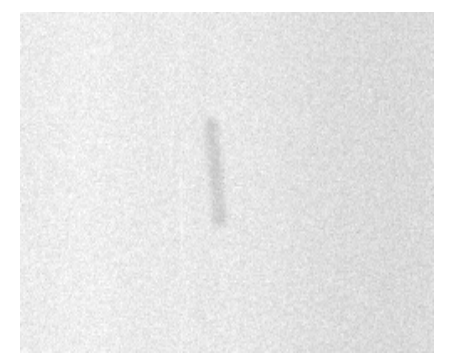

(b)Contrast enhancement

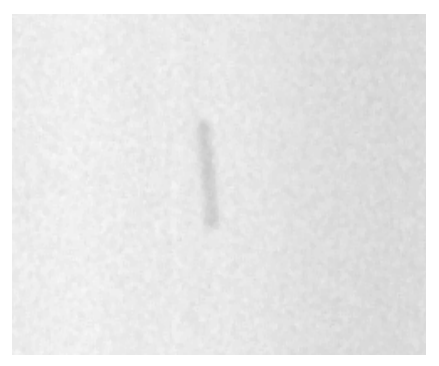

(c)Median filtering

Fig.2 Results of image preprocessing 


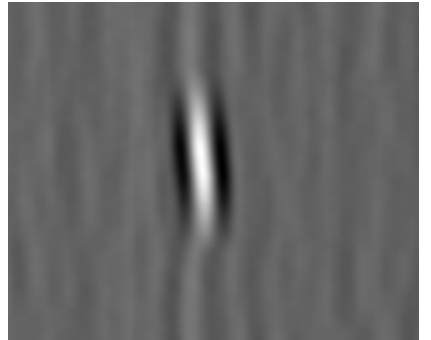

(d)Gabor matched filtering

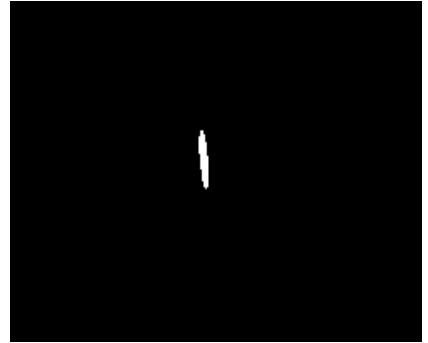

(e)Binarization

Fig.3 Results of Gabor filtering and image binarization

\section{Conclusions}

In this paper, based on the analysis of high-speed small target in large field of view, the Gabor convolution kernel is designed based on the image texture features at time axis and space axis. An image processing algorithm is proposed to detect small targets in large field of view. Firstly, the image preprocessing is done by grayscale adjustment and median filtering. The image contrast is enhanced and the image random noise is removed. Then the Gabor filter is used to extract the small target texture, and then the image is binarized .The algorithm is implemented in MATLAB. Finally, the algorithm is used to detect the small target in large field of view in a practical experiment, and the algorithm is proved to be effective.

\section{References}

[1] Li Mingjing. Concept research on stations arrangement of active measurement system for more, small, rapid and dark objects[D].Chinese Academy of Science, China(Changchun Institute of Optics, Fine Mechanics and Physics),2011.

[2] Niu Shaoqing.Study of infrared small and weak target detection in complex background[D]. Huazhong University of Science \& Technology ,2013.

[3] Xue Lixia, Luo Yanli, Wang Zuocheng. Detection algorithm of adaptive moving objects based on frame difference method[J]. Application Research of Computers,2011(4).

[4]A D J, Berlintozzi A L.A wavelet-laplace variational technique for image deconvolution and impainting[J].IEEE Transactions on Image Processing,2008,17(5):657-663.

[5] Liu Yuan, Tang Xinyi, Li Zheng. A new top hat local contrast based algorithm for infrared small target detection [J]. Infrared Technology, 2015, 37(7):544-552.

[6]Yang Lei. Study on infrared small target detection and tracking algorithm under complex backgrounds[D].Institute of Image Processing and Pattern Recognition, Shanghai Jiao Tong University,2006.

[7]Cao Yuan, Yang Jie, Liu Ruiming. Detecting infrared small target by using TDLMS filter based on neighborhood analysis[J].Journal of Infrared and Millimeter Waves, 2009,28(3):235-240.

[8]Wu Jiangpeng, Song Ping, Hao Chuangbo, Li Zhida. Method for measuring space-time distributed parameters of multiple projectiles[J].ACTA Armamentarii, 2015,36(10):1967-1974.

[9] Luo Xiaoqing, Wu Xiaojun. Detection algorithm for infrared small and weak targets based on wavelet transform and Gabor filter[J]. Infrared and Laser Engineering, 2011, 40(9):1818-1822.

[10] YAN Haiting, WANG Ling, LI Kunming, et al. Face recognition based on Gabor Directional Pattern[J].Computer Engineering and Applications, 2015, 51(10):166-169.

[11] Huang Zhanhua, Liu Zheng ,LiJinjing, Kong Mao, Nie Shoushuai,Cai Huaiyu.Texture extracting approach on mused Gabor filters[J].ChineseJournalofScientificInstrument,2009,30(4): 694-698. 